Regular Articles

\title{
Comment on " $\mathrm{GHz}$ pulse train generation in fiber lasers by cavity induced modulation instability"
}

\author{
Stefan Wabnitz* \\ Dipartimento di Ingegneria dell'Informazione, Università degli Studi di Brescia, via Branze 38, 25123 Brescia, Italy
}

\section{A R T I C L E I N F O}

Article history:

Received 13 December 2014

Revised 22 May 2015

Available online 13 July 2015

Keywords:

Mode-locked lasers

Ultrafast fiber laser

Modulation instability

Nonlinear fiber optics

\begin{abstract}
A B S T R A C T
A recent invited paper (Tang et al., 2014) reports the experimental observation of the generation of stable pulse trains in a ring fiber laser with repetition rates varying in the range between $3 \mathrm{GHz}$ and $285 \mathrm{GHz}$. Contrary to what is stated, the theory published in that invited paper does not support the claim that the generation mechanism of the pulse train is the cavity induced modulation instability effect.
\end{abstract}

(c) 2015 Elsevier Inc. All rights reserved.
In a recent invited paper [1], Tang et al. describe a fiber ring laser comprising an erbium doped fiber pumped by a $1480 \mathrm{~nm}$, high power Raman fiber laser, a span of standard single mode fiber, a polarization controller (PC) and a polarization-independent isolator. The average cavity dispersion is anomalous and equal to about $3.3 \mathrm{ps} / \mathrm{nm} / \mathrm{km}$. By increasing the pump power above $200 \mathrm{~mW}$, and by suitably adjusting the intracavity PC, the cw mode of operation of the laser became unstable, and break-up of the cw emission into a train of sub-picosecond pulses with repetition rates continuously varying in the range between $3 \mathrm{GHz}$ [see Fig. 3(a) of Ref. [1]] and $32 \mathrm{GHz}$ [see Fig. 4(a) of Ref. [1]], depending on the PC waveplates orientation, was observed. On the other hand, for a given PC configuration and by increasing the pump power up to $2 \mathrm{~W}$, the repetition rate of the generated pulse train increased up to about $285 \mathrm{GHz}$ [see Fig. 5(a) of Ref. [1]]. Clearly, the particular setting of the intracavity PC determines the net cavity birefringence of the fiber laser. The cavity birefringence of their fiber laser was not estimated nor measured by Tang et al.; however they have stated on page 612 of Ref. [1] that the linear birefringence of the cavity is somehow related to the cavity detuning, although this relationship is never expressed in explicit form. In fact, in describing their pulse train generation experiments, Tang et al. use the term "cavity detuning" as being equivalent to "cavity birefringence".

Based on the observation that the observed pulse repetition rate depends on "cavity birefringence", and on their hypothesis that "cavity birefringence" is equivalent to "cavity detuning", Tang et al. claim that the explanation of their experiments is based on

\footnotetext{
* Fax: +39030380014.

E-mail address: stefan.wabnitz@unibs.it
}

the cavity modulation instability effect which was described in Refs. [2-4]. In fact, the "Theoretical description" section of Ref. [1] repeats the analysis of the modulational instability that is induced by cavity boundary conditions in a cw pumped, passive nonlinear cavity that was presented in Ref. [2] (see also the original Ref. [5] for the formally identical transverse instability).

However the laser cavity that was described in Ref. [2] is fundamentally different from the fiber ring laser presented in Ref. [1]. As a matter of fact, Ref. [2] describes a passive fiber ring cavity pumped by an external $\mathrm{cw}$ laser beam. In that situation, the cw field builds up in the cavity (of length $\mathrm{L}$ and with linear refractive index $n$, possibly including an intensity dependent contribution) at the same frequency of the external pump laser, say, $\omega_{0}$, until a steady intracavity power level is reached. Remarkably, the pump laser frequency $\omega_{0}$ does not need to exactly match a particular resonance or longitudinal mode of the passive fiber cavity, say, $\omega_{R}[2-4]$. This leads to a nonzero cavity detuning $\delta=\left(\omega_{\mathrm{R}}-\omega_{0}\right) t_{\mathrm{R}}$ between the linear phase delay at the pump laser frequency $\omega_{0} t_{R}=\omega_{0} n \mathrm{~L} / \mathrm{c}$ (equal to $\psi_{0}$ in the notation of Ref. [1], see Eq. (2)], and the linear phase delay at a nearby cavity resonance frequency $\omega_{R}$, namely $\omega_{R} t_{R}=2 \mathrm{~m} \pi$, with integer $\mathrm{m}$. To the contrary, in a fiber ring laser with no externally injected pump beam, such as the laser that is discussed in Ref. [1], the cw field is necessarily locked at a given cavity frequency $\omega_{R}$, as it is well known from basic laser theory [6].

Therefore the linear phase delay $\psi_{0}$ in the case of the laser described in Ref. [1] is a fixed quantity, and not a free parameter that can be arbitrarily varied as in the examples provided in Fig. 1 of Ref. [1]. Indeed, no control of the cavity phase detuning is possible in the laser of Ref. [1], contrary to the claims by its Authors. 
Coming back to the other claim by the Authors of Ref. [1], namely that the cavity detuning of their laser can be controlled by varying the cavity birefringence, it is well known that by varying the net cavity birefringence one may shift the cavity resonance, but it is not possible to drive the cw laser out of resonance.

As far as the possible explanation of the wide range of variation of the observed modulational instability frequency as a function of the cavity birefringence, we may suggest the modulational polarization instability effect [7], which is known to have a gain that extends all the way down to zero modulation frequencies for sufficiently low net birefringence, and whenever the fast axis of the fiber is pumped. Moreover, it is well known that self-pulsations and mode-locking may occur in ring fiber lasers via the mechanism of dissipative four-wave mixing [8], independently of the presence (or lack thereof) of modulation instability [9]. Therefore, for a proper interpretation of the experimental results of Ref. [1], it might be suitable to develop a vector theory of dissipative four-wave mixing, including the action of cavity birefringence.

\section{References}

[1] D.Y. Tang, J. Guo, Y.F. Song, L. Li, L.M. Zhao, D.Y. Shen, GHz pulse train generation in fiber lasers by cavity induced modulation instability, Opt. Fiber Technol. 20 (2014) 610-614.

[2] S. Coen, M. Haelterman, Modulational instability induced by cavity boundary conditions in a normally dispersive optical fiber, Phys. Rev. Lett. 79 (1997) 4139-4142.

[3] M. Haelterman, S. Trillo, S. Wabnitz, Dissipative modulation instability in a nonlinear dispersive ring cavity, Opt. Commun. 91 (1992) 401-407.

[4] M. Haelterman, S. Trillo, S. Wabnitz, Additive-modulation-instability ring laser in the normal dispersion regime of a fiber, Opt. Lett. 17 (1992) 745-747.

[5] D.W. McLaughlin, J.V. Moloney, A.C. Newell, New class of instabilities in passive optical cavities, Phys. Rev. Lett. 54 (1985) 681-684.

[6] A. Yariv, Quantum Electronics, third ed., J. Wiley, New York, 1989, p. 187.

[7] S. Wabnitz, Modulational polarization instability of light in a nonlinear birefringent dispersive medium, Phys. Rev. A 38 (1988) 2018-2021.

[8] M. Quiroga-Teixeiro, C. Balslev Clausen, M.P. Sørensen, P.L. Christiansen, P.A Andrekson, Passive mode locking by dissipative four-wave mixing, J. Opt. Soc Am. B 15 (1998) 1315-1321.

[9] T. Sylvestre, S. Coen, P. Emplit, M. Haelterman, Self-induced modulational instability laser revisited: normal dispersion and dark-pulse train generation, Opt. Lett. 27 (2002) 482-484. 